\title{
Full scale monitoring of wind and traffic induced response of a suspension bridge
}

\author{
Etienne Cheynet ${ }^{1, a}$, Jasna Bogunović Jakobsen ${ }^{1}$, and Jónas pór Snæbjörnsson ${ }^{1,2}$ \\ ${ }^{1}$ Department of Mechanical and Structural Engineering and Materials Science, University of Stavanger, Norway \\ ${ }^{2}$ School of Science and Engineering, Reykjavik University, Iceland
}

\begin{abstract}
This paper presents a full-scale analysis of wind and traffic-induced vibrations of a long-span suspension bridge in complex terrain. Several wind and acceleration sensors have been installed along the main span on Lysefjord Bridge in Norway. In the present study, three days of continuous records are analysed. Traffic-induced vibrations are dominant at low and moderated wind speed, with non-negligible effects on the overall bridge response for heavy vehicles only. Traffic and wind-induced vibrations are compared in terms of root mean square of the acceleration response, and three simples approaches are proposed to isolate records dominated by wind-induced vibration. The first one relies on the separation of nocturnal and diurnal samples. The second one is based on the evaluation of the time-varying root mean square of the acceleration response. The last one evaluates the relative importance of the high frequency domain of the acceleration bridge response. It appears that traffic-induced vibrations may have to be taken into account for the buffeting analysis of longspan bridge under moderated wind.
\end{abstract}

\section{Introduction}

Relatively few analyses of wind and traffic-induced vibrations of full-scale suspension bridges in complex terrain have been done. Hui $[1,2]$ did a detailed analysis of the wind conditions near the Stonecutter Bridge, which is subjected to both a sea and mountain wind, by using a wind mast. Macdonald [3] studied the response to wind and traffic load of the Clifton Bridge, which is a relatively shortspan suspension bridge crossing a narrow gorge. Cai [4] proposed a detailed analytical model to study both wind and traffic induced vibrations of a long span bridge, but no detailed measurement campaign of such phenomenon has been found, apart from Macdonald [3]. In Norway, the coastal highway E39 project requires a detailed description of the wind conditions and bridge response in complex topography such as fjords. Long span bridges already in service are ideal study cases for such purpose, but traffic induced vibrations may perturb the buffeting analysis. Heavy trucks crossing the bridge are likely to produce non-negligible vibrations, but they are often ignored. This assumption is likely to be justified for strong winds only. The present paper investigates the influence of traffic on wind-induced vibrations of the Lysefjord Bridge in Norway. First the topography and the wind conditions observed at Lysefjord are described. Then the influence of traffic-induced vibrations is highlighted using the peak and standard deviation of the acceleration response. Finally, three strategies are proposed to separate records domi-

${ }^{\mathrm{a}}$ Corresponding author: etienne.cheynet@uis.no nated by traffic-induced vibrations from those affected by wind turbulence only.

\section{Bridge site and instrumentation}

The Lysefjord Bridge (figure 1) crosses the narrow inlet of a fjord in the South-West of Norway, at an altitude of 55 $\mathrm{m}$. Its main span is $446 \mathrm{~m}$ long and is oriented from NorthWest to South-East. Steep hills and high cliffs surround its extremities. Its East side is oriented toward the inside of the fjord while its West side faces a more open area.

The proximity of the mountains and small islands is likely to be responsible for a very high turbulence intensity. The channelling effect of the fjord produces local wind conditions that requires the installation of wind sensors directly on the bridge. At the beginning of October 2014, seven sonic anemometers and four couples of tri-axial accelerometers were operational along the bridge deck (figure 2). The accelerometers are installed near Hangers 09, 18, 24 and 30 on both side of the deck so that lateral, vertical and torsional accelerations can be recorded. The anemometers located on hangers 10, 16, $18,20,24$ and 30 are placed about $6 \mathrm{~m}$ above the West side of the deck. Two additional anemometers are installed on hanger 08 . They are noted H- $08 \mathrm{~b}$ and $\mathrm{H}-08 \mathrm{t}$ and are located 6 and $10 \mathrm{~m}$ above the bridge deck respectively. The anemometer on H-10 is a Waisala weather transmitter WXT520 while the other ones are 3D WindMaster Pro sonic anemometers. Acceleration and wind data are synchronized using GPS timing and down-sampled to $20 \mathrm{~Hz}$. 
The recording is continuous over every 10 minutes and the files are transferred using a mobile net.
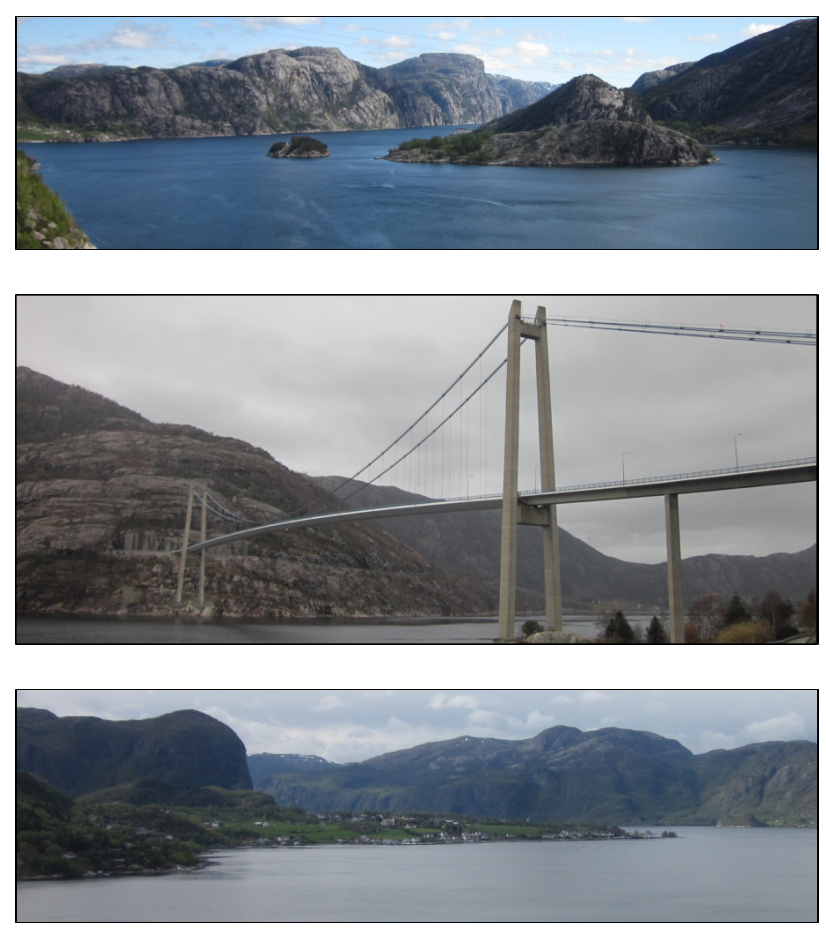

Figure 1. View to the North-East (top), the bridge (middle) and view to the South-West (bottom) from the bridge.

The wind components normal and along the bridge deck refer to the "bridge-based" coordinate system and are denoted $V_{x}+v_{x}$ and $V_{y}+v_{y}$ respectively. These components are split into a mean part $\left(V_{x}\right.$ and $\left.V_{y}\right)$ and a fluctuating one $\left(v_{x}\right.$ and $\left.v_{y}\right)$. The along and across-wind components, noted $U+u$ and $v$, are recovered by projecting the wind components in the bridge-based coordinate system onto the mean wind direction, at the yaw angle $\beta$, and normal to it. Similarly, $u$ and $v$ denote the fluctuating components, and $U$ denotes the mean components.

Two main wind conditions are usually observed at Lysefjord Bridge. On one hand, the wind from N-NE comes from the inside of the fjord, with a high turbulence intensity. On the other, the wind from S-SW "hits" the bridge before entering into the fjord, with usually a higher wind speed than the wind from N-NE. In the present study, three days of wind and acceleration data from the $01 / 10 / 2014$ to the $03 / 10 / 2014$ are selected. The usual wind conditions are not fully verified here, because of the low wind speed recorded (figure 3), which allows nevertheless the investigation of the influence of traffic-induced vibrations.

It has been observed that the two main wind direction lead to two distinct wind conditions, with different spatial and temporal characteristics. Consequently, the buffeting response of the bridge is studied by using a case by case approach, i.e. by separating the wind from $S$ SW and N-NE. When the wind direction changes, a nonstationary wind field is usually recorded. However, the wind induced non-stationary response is generally easily distinguished from traffic-induced vibrations, that occur at a much smaller time scale.

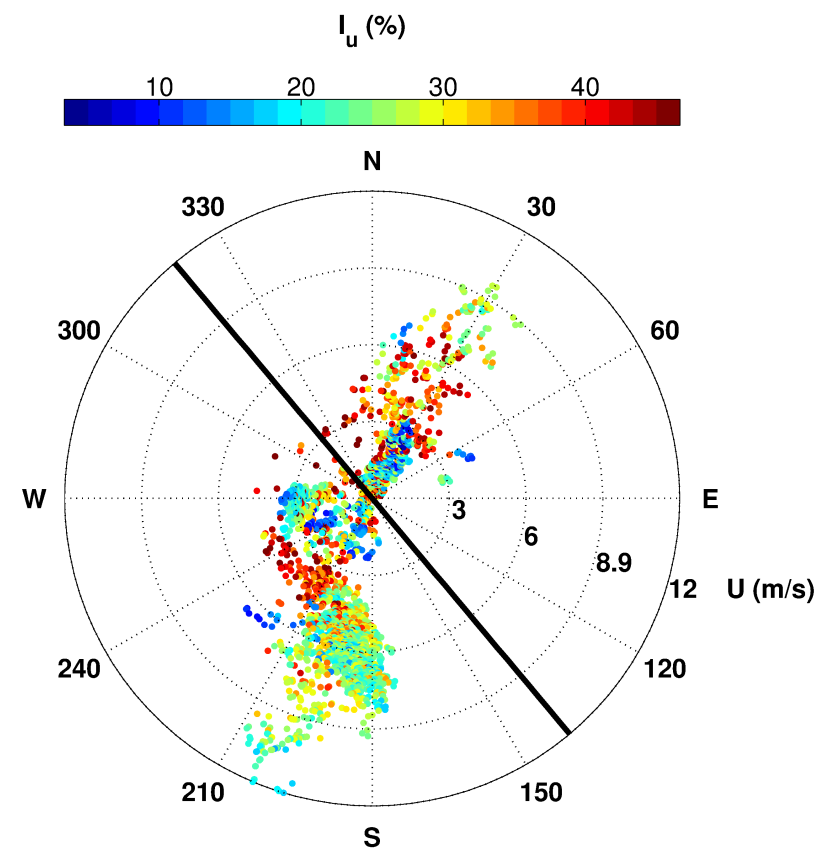

Figure 3. Mean wind speed and turbulence intensity as a function of the wind direction from $01 / 10 / 2014$ to $03 / 10 / 2014$.

\section{Separation of wind and traffic-induced vibrations}

The buffeting response of Lysefjord Bridge can be studied by separating samples recorded during the night and the day, by assuming that no heavy vehicles cross the bridge at night-time. The definition of day-time and night-time is arbitrary. It can be done by comparing the periodicity of the bridge vibration during several days that are characterized by a very low wind speed. The day-time is started on the morning when the bridge response start increasing while the mean wind speed remains unchanged. The night-time is started when the response of the bridge dramatically decrease at the end of the day. One drawback of the method is the reduction of the number of available samples, which is already lowered by using a case by case approach to separate wind from N-NE and from S-SW. However, this is the most simple and straightforward method to reduce the number of samples affected by traffic.

Another method is to consider only samples with a high mean wind speed. Macdonald [3] noted that an appropriate threshold wind speed for the Clifton suspension bridge may be $8 \mathrm{~m} / \mathrm{s}$. For the Lysefjord Bridge that limit is likely to be different. One of the purpose of this paper is to look at the bridge acceleration response with a low wind speed, and therefore this method is not applied here.

Another possibility to eliminate records dominated by traffic-induced vibrations is to study the non-stationarity 


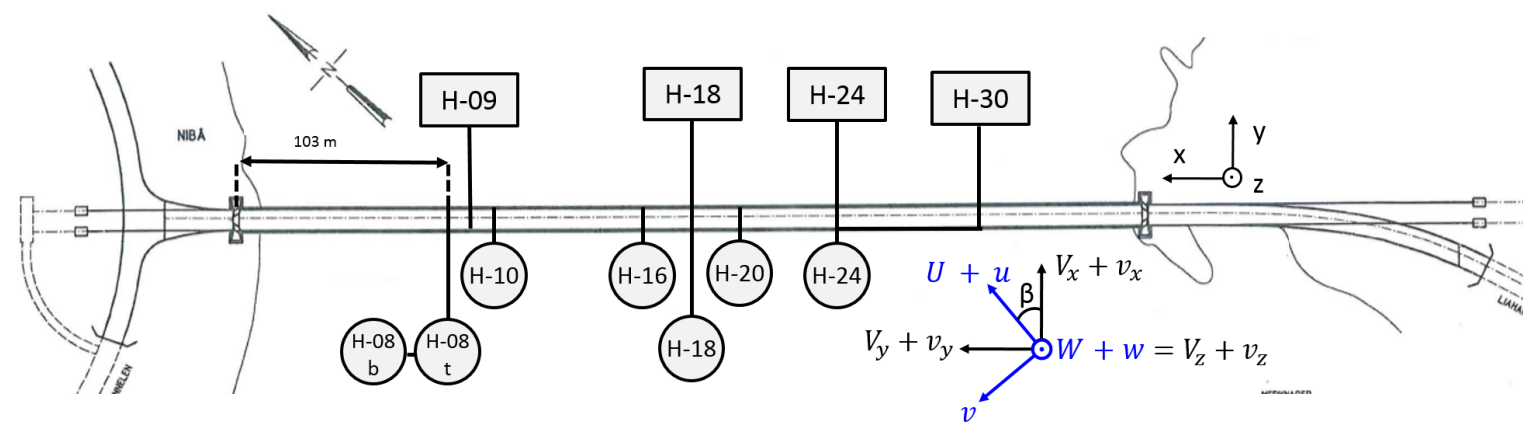

Figure 2. Anemometers (circles) and accelerometers(rectangles) installed on Lysefjord Bridge

of the bridge response. The goal is to find an exclusion criterion that dismisses acceleration records influenced by heavy vehicles. This criterion relies on the time varying standard deviation of the vertical bridge acceleration response. Every single 10-minutes record is divided into 300 segments of 2 seconds, which mean that the slowest frequency recorded in each segment is above the first vertical symmetric and asymmetric eigen-frequency of the bridge deck that are equal to 0.29 and $0.21 \mathrm{~Hz}$ respectively. For every 2 -seconds segment, the time varying standard deviation $\sigma_{A_{z}}(t)$ of the bridge acceleration response at mid-span is calculated. However, variations of $\sigma_{A_{z}}(t)$ can be due to traffic or wind gusts. The time derivative of the turbulent wind component $w$ is defined as :

$$
\dot{w}=\frac{\partial w}{\partial t}
$$

The variation rate $\tau$ of the vertical bridge acceleration is used as exclusion criteria and defined using the standard deviation of $\dot{w}$ written $\sigma_{\dot{w}}$ :

$$
\tau=\frac{\sigma_{A_{z}}(t)}{\sigma_{\dot{w}}(t)}
$$

A sudden variation of the wind speed is likely to lead to a high value for $\sigma_{\dot{w}}$, and therefore a lower value for $\tau$. Consequently, high values of $\tau$ are reached only when strong traffic-induced vibrations are recorded. One limitation is that if a strong wind gust occurs while a heavy vehicle is crossing the bridge, then $\tau$ is likely to be abnormally low. If the lateral bridge response is studied, the turbulent component $u$ should be used instead of $w$.

As underlined by Macdonald [3], traffic-induced vibrations affect mainly the high frequency content of the bridge response. It should be possible to detect any nonnegligible traffic-induced response by comparing the low and high frequency part of the power spectral density (PSD) of the vertical bridge acceleration response, written $S_{A_{z}}$. In the following, the separation between the high and low frequency content is arbitrary set to $4 \mathrm{~Hz}$, while the Nyquist frequency is fixed to $10 \mathrm{~Hz}$. The relative contribution of the frequency interval $\left[f_{1}, f_{2}\right]$ to the RMS of the acceleration response is written $\sigma_{f_{1}-f_{2}}$, and defined as

$$
\sigma_{f_{1}-f_{2}}=\sqrt{\int_{f_{1}}^{f_{2}} S_{A_{z}}(f) d f}
$$

Therefore, another criteria $\lambda$ can be defined as the ratio between the high and low frequency contribution to the RMS of the bridge response:

$$
\lambda=\frac{\sigma_{4-10}}{\sigma_{0-4}}
$$

If the criteria $\lambda$ is higher than 1 , then the bridge acceleration response is assumed to be dominated by traffic induced vibration

\section{Results and discussions}

The acceleration and wind records from the 01/10/2014 to the $03 / 10 / 2014$ are studied in the following. On figure 5 , a periodic fluctuation of the vertical peak response is observed. A much higher response is recorded during the day than during the night, and was also observed by Macdonald [3]. The corresponding wind records shows that the bridge response is not always correlated with the mean wind speed. On 02/10/2014, the wind speed was lower than $3 \mathrm{~m} / \mathrm{s}$, but the peak response still increases during the day. When such a low wind speed is recorded, the wind direction tends to change often. During the period of study, the N-NE wind was mainly recorded during the night, while the S-SW wind was dominant during the day. Strong variations of the peak response are likely to be due to short duration events like traffic-induced vibrations. The peak response may also be higher than usual when the wind direction changes from N-NE to S-SW. In that case, the wind field may become temporally non-stationary and characterized by relatively strong wind gusts. However, the mean wind speed may have not have been high enough to make such a phenomenon visible on figure 5 .

A selected acceleration response, recorded between 06:50 and 07:00 on 02/10/2014, is displayed on figure 4 . The lateral, vertical and torsional response are written $A_{x}$, $A_{z}$ and $A_{t}$ respectively. The mean wind speed is $1.2 \mathrm{~m} / \mathrm{s}$ and the turbulence intensity for the $\mathrm{u}$ and $\mathrm{w}$-component is $18 \%$ and $6 \%$ respectively. At very low wind speed, 

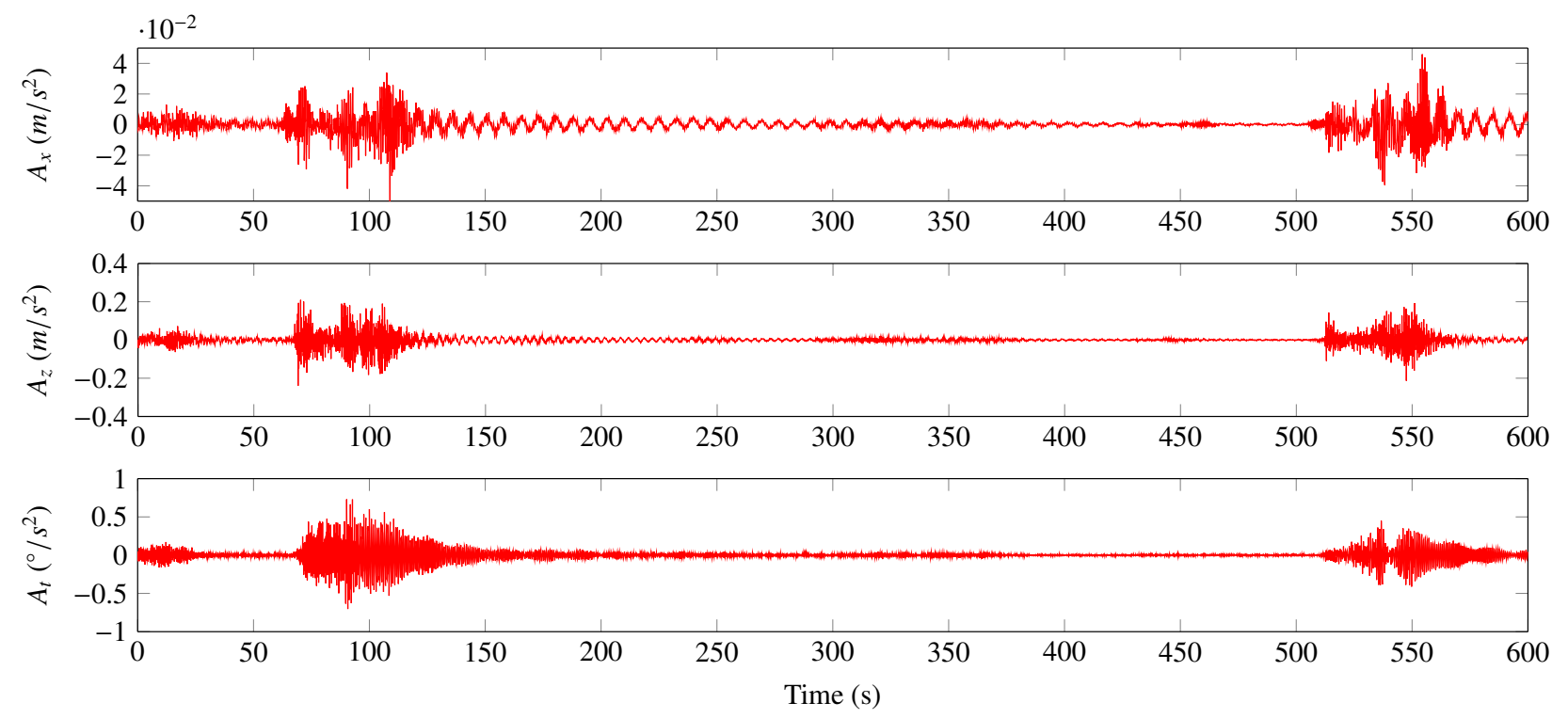

Figure 4. Lateral, vertical and torsional acceleration at mid-span on Lysefjord Bridge on 02/10/2014 between 06:50 and 07:00

the turbulence intensity is expected to be much higher [5]. The bridge response to wind turbulence is nearly nill at this time, and the main source of vibrations is due to traffic.

On figure 4, the vertical response is considerably more affected than the lateral one, which is to be expected. Two important peaks, likely due to heavy vehicles, are observed, at about 100 and $540 \mathrm{sec}$. The lowest frequency of interest ranges from 20 to $30 \mathrm{mHz}$, and corresponds to the inverse of the time required for the truck to cross the main span. Unfortunately, the accelerometers installed inside the bridge deck are not designed to accurately record vibrations under $60 \mathrm{mHz}$, which prevents a detailed analysis of this phenomenon.

When a heavy truck enters the bridge main span, an impulse is first recorded by the accelerometers. While the truck crosses the main-span, every eigen-frequency of the bridge are excited, but with a higher energy content for the high frequency domain (higher than $4 \mathrm{~Hz}$ ) than observed for wind-induced excitations. It explains why the acceleration records on figure 4 appear noisy when traffic-induced vibration is recorded. Such vibrations are non-stationary and may be interpreted as a transient bridge response by contrast to wind-induced vibrations that are generally assumed to be stationary, at least for neutral wind, and wind velocities above $8 \mathrm{~m} / \mathrm{s}$.

These observations are highlighted by the comparison of two spectrograms of the vertical bridge response on figure 6. The left one is based on an acceleration record without traffic, and the right one shows important trafficinduced vibrations. For the left figure, it can be assumed that only the first three symmetric eigen-modes are excited, which corresponds to a frequency of 0.29 and 0.41 , and $0.86 \mathrm{~Hz}$ respectively. The amplitude remains almost constant with time. It is clearly not the case for the right panel, where much more eigen-modes are excited. The amplitude of the higher modes decays rapidly with the time, while the decay of the lower ones is slower but nonnegligible.
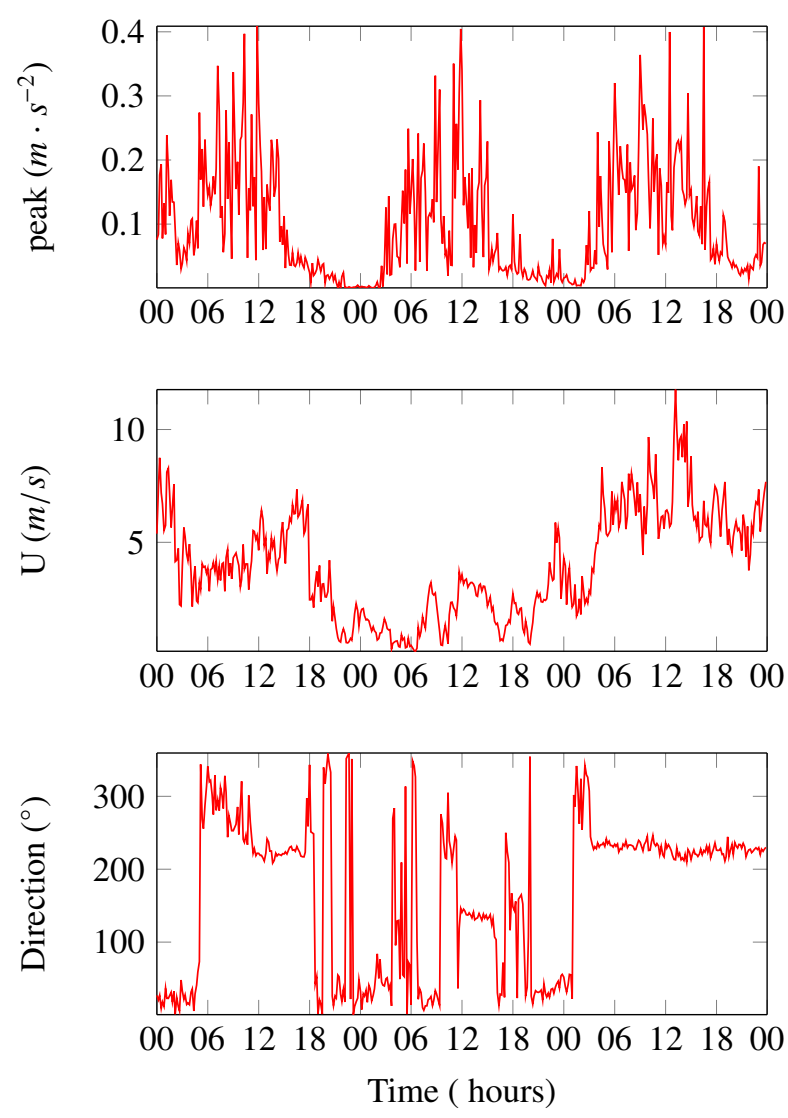

Figure 5. Peak acceleration response (top), mean wind speed recorded (middle), and mean wind direction (bottom) at mid-span from $01 / 10 / 2014$ to $03 / 10 / 2014$. 


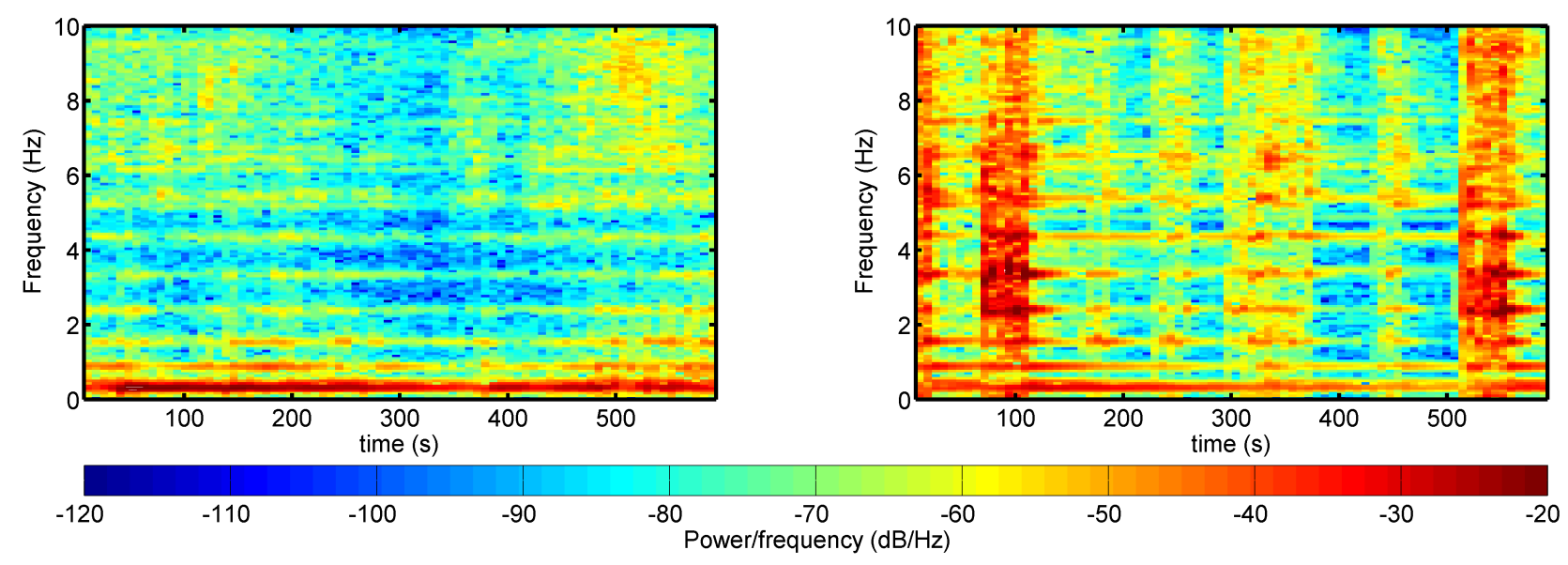

Figure 6. Spectrogram of the vertical bridge acceleration response at mid-span, on 02/10/2014 between 00:00 and 00:10 (left) 06:50 and 07:00 (right).

In the time series from figure 4, only small amplitude vibrations are recorded around $t=150 \mathrm{~s}$ and $t=500 \mathrm{~s}$. The right spectrogram from figure 6 shows that several small vehicles have probably crossed the bridge. However, their influence on the bridge response is likely to be negligible. The comparison of the first eigen-frequencies of the bridge deck for the records from figure 4 and from a sample recorded on the previous night did not show any visible modification of the eigen-frequencies of the bridge due to heavy trucks, as it was observed by Kim [6].

Macdonald [3] underlines that traffic-induced vibrations mainly excite the high frequency components of the response spectrum, but the spectrogram of a 10-minutes records in figure 6 shows that every frequency within the range analysed is actually excited. For a period of 10 minutes, it confirms also that the bridge response under traffic load is not stationary, since the response spectrum shows strong variations with time. The low frequency part of the spectrogram may be considered as stationary for shorter periods ranging from one to two minutes. Consequently, filtering cannot be applied to separate the traffic and wind-induced vibrations, because every frequency of the response spectrum is affected to some degree.

\subsection{Segregation using diurnal and nocturnal data}

Using figure 5, the night-time is selected as the period between 18:00 and 05:00. Wind from N-NE is observed in the present study mainly during the night. A N-NE wind leads to a larger spreading of the RMS of the bridge response $\sigma_{z}$, even at night-time (figure 7). According to the buffeting theory [7], different turbulence intensities lead to different bridge responses for a given mean wind speed. The N-NE wind shows a stronger variability than the SSW winds. The spread out of the RMS of the acceleration response can be reduced if divided by the underlying turbulence intensity and fitted by an empirical power law with a power coefficient ranging from 2 to 3 [3]. If the buffeting theory is well verified, the RMS of the bridge acceleration should agree well with the power law, while data dominated by traffic-induced vibrations should show a strong discrepancy. For a N-NE wind, the diurnal records tend to correspond mainly to samples dominated by trafficinduced vibrations, since they disagree with the power law described previously.

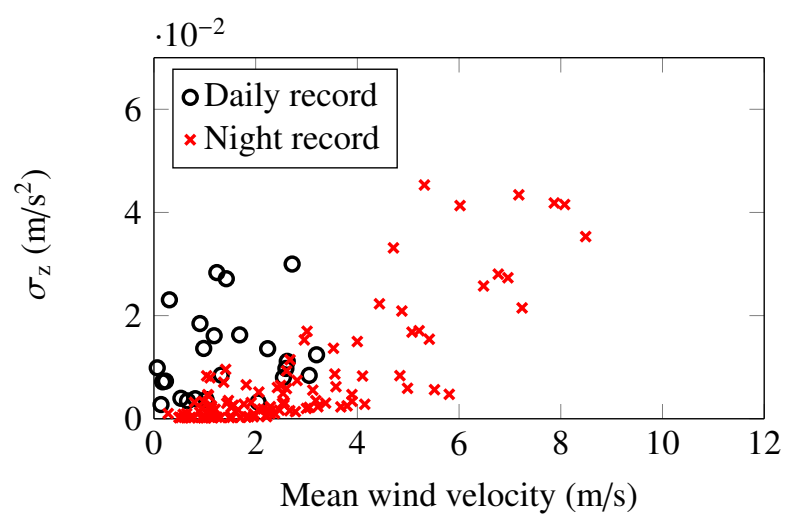

Figure 7. Diurnal and nocturnal separation (N-NE wind).

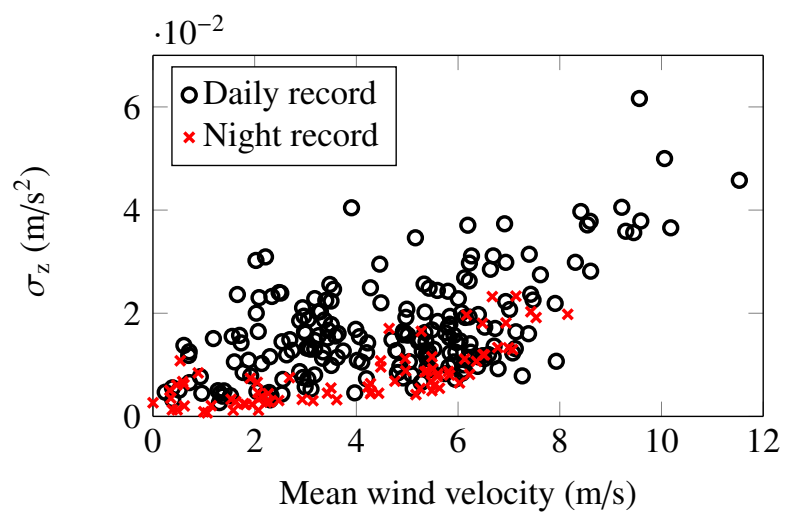

Figure 8. Diurnal and nocturnal separation (S-SW wind). 
For the S-SW wind (figure 8), the diurnal samples are clearly affected by traffic-induced vibration. However, the wind-induced response seems to dominate most of the records, as the general trend follows the power law, particularly for the higher wind velocities as is to be expected. Therefore, the simple approach of separating diurnal and nocturnal samples may cause the loss of valuable windinduced response data for S-SW winds.

The choice of a segregation criterion may be preferred to the use of nocturnal records. Nevertheless, it is verified based on these diurnal and nocturnal data. The validation procedure is done using samples recorded for strong winds, that are assumed to be the only source of vibrations, i.e. traffic has become negligible.

\subsection{Segregation using the response variability}

The threshold value of $\tau$ beyond which traffic-induced vibration is assumed to be dominant for the vertical bridge response is arbitrary chosen. For a N-NE wind, it lays most of the time below a value of 0.1 . In the following, a threshold value of 0.025 is chosen. This threshold depends also on the length of the segments used to calculate $\sigma_{A_{z}}$. The susceptibility of the lateral bridge response to traffic is lower than for the vertical response, leading to a lower value for $\tau$. Macdonald [3] observed that at high wind speed, the maximal RMS of the acceleration response of the Clifton suspension bridge was broadly 4 times higher than the maximum traffic response. On figure 11 and 12, only the vertical response is considered.

For a N-NE wind, little difference is observed between figure 9 and 7 for low wind speeds. Some nocturnal records are however counted as dominated by trafficinduced vibrations. For a S-SW wind, much greater number of records are counted as "traffic-free". Some samples displaying a high RMS at low wind speed are not counted as dominated by traffic-induced vibration. One reason may be that the threshold value for $\tau$ is too low. A more time consuming task to select samples dominated by wind-induced vibrations would be to manually look at the acceleration time series.

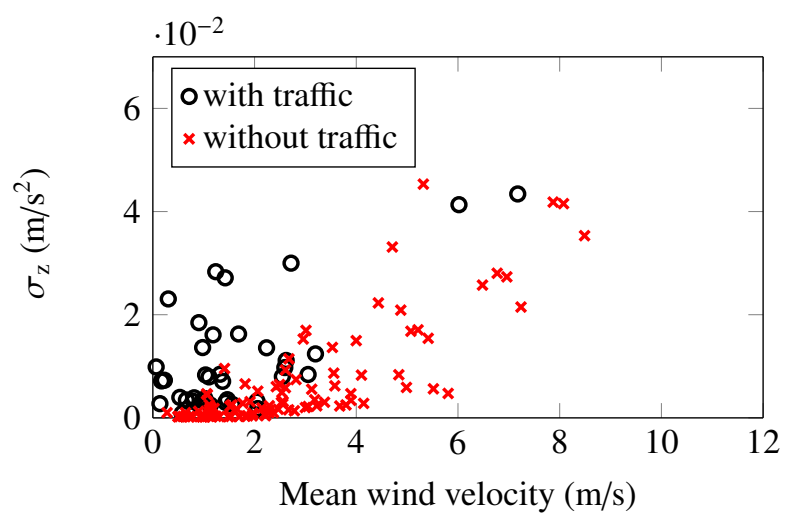

Figure 9. RMS of vertical bridge acceleration at mid-span for a N-NE wind using the criteria $\tau$.

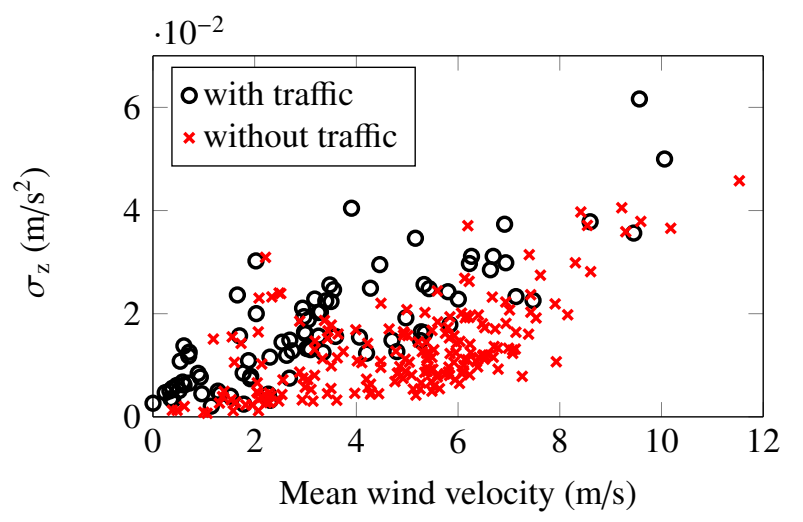

Figure 10. RMS of vertical bridge acceleration at midspan for a S-SW wind using the criteria $\tau$.

More generally, the criteria $\tau$ often highlights higher response amplitudes for a given wind speed, which is to be expected. For high wind speeds, the response variability criterion often misinterprets strong wind gusts as trafficinduced vibrations, unless the value of $\tau$ is increased. It may reduce its application to low and moderate wind speeds only. For this reason, another criterion based on the description of the bridge response in the frequency domain is proposed.

\subsection{Segregation using the response spectral density}

The separation of traffic and wind-induced vibration by comparing the low and high frequency content of the PSD of the bridge response requires stationary data. It si not the case when traffic is dominant for a 10-minutes long record. The shortest length for which the records are assumed stationary is set to 30 seconds, which is approximately the time vehicles spend to cross the main span. The PSD of the bridge response is consequently calculated for a minimal frequency of $33 \mathrm{mHz}$. If one of the 30 -seconds segment is detected as dominated by traffic-induced vibrations, then the whole 10-minute samples from which the segment comes from is dismissed.

The application of the criteria $\lambda$ is presented for a $\mathrm{N}$ NE wind on figure 11 and for a S-SW one on figure 12. A good agreement with the nocturnal and diurnal samples is obtained. The criteria $\lambda$ may overestimate the number of samples dominated by traffic at low wind speed. For strong winds, $\lambda$ detects almost no traffic-induced vibration, as is to be expected. The criterion $\tau$ may be more adapted for low wind speeds, while $\lambda$ seems more reliable for high wind speeds.

The low wind speed recorded during these three days does not allow an accurate estimation of the threshold mean wind speed beyond which every type of trafficinduced vibration becomes negligible. The analysis of several samples from a N-NE wind with a mean wind speed up to $16 \mathrm{~m} / \mathrm{s}$ has shown that nocturnal and diurnal samples did not show strong differences above $12 \mathrm{~m} / \mathrm{s}$. 


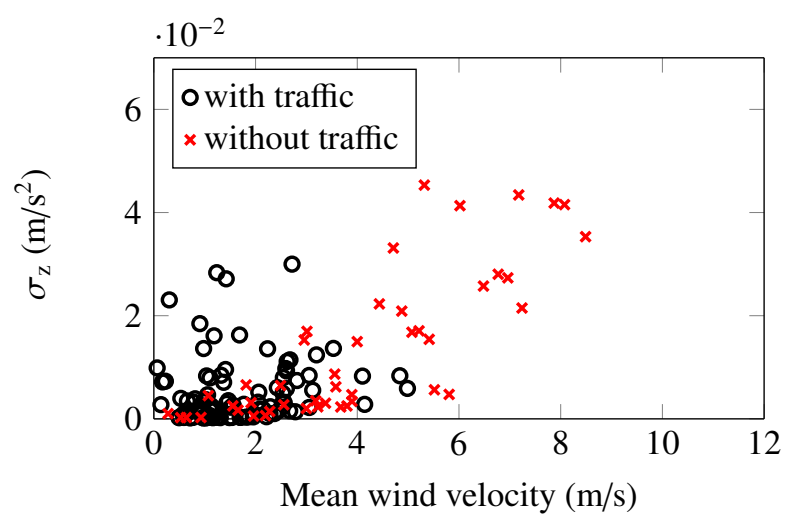

Figure 11. RMS of vertical bridge acceleration at midspan for a N-NE wind using the criteria $\lambda$.

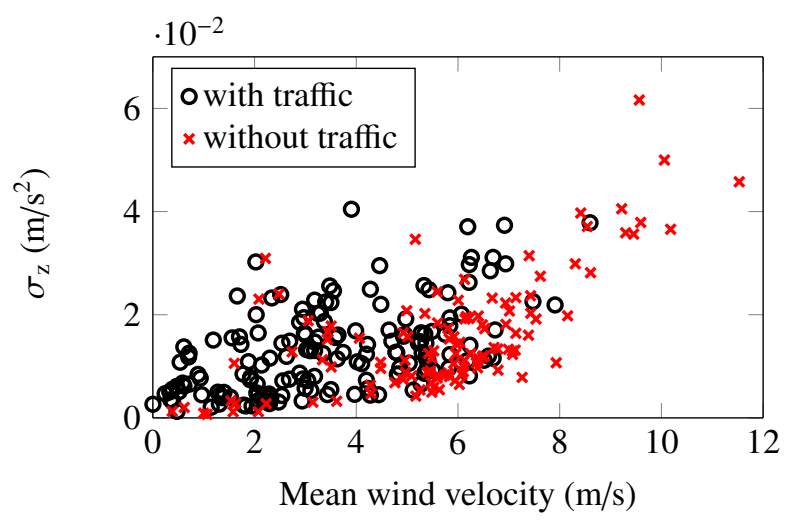

Figure 12. RMS of vertical bridge acceleration at midspan for a S-SW wind using the criteria $\lambda$.

\section{Conclusion}

The analysis of acceleration records of the Lysefjord bridge shows that heavy vehicles are responsible for nonnegligible vibrations. The buffeting analysis of long-span suspension bridges is likely to be affected by such parasitic phenomena. Therefore, three simple approaches to remove samples dominated by traffic-induced vibrations are proposed:

(1) The separation of diurnal and nocturnal records.

(2) The application of the criterion $\tau$, based on the timevarying standard deviation of the bridge acceleration response.

(3) The application of the criterion $\lambda$, based on the relative importance of the high frequency components of the acceleration response.
The selection of nocturnal records is straightforward, but may be too harsh. For this reason, the criterion $\tau$ is proposed, and may efficiently detects variation of the acceleration response due to heavy vehicles, but at low and moderated wind speeds only. Another criterion $\lambda$ may be more reliable for strong winds, but tends to overestimate the number of samples dominated by traffic-induced vibration at low wind speed. Alternative forms of these criteria will be further explored.

In June 2015, one GPS sensor was installed on the middle of the Lysefjord bridge. The direct measurement of the bridge displacements may bring more information about its low frequency behaviour and should help to track the displacement of the bridge under a heavy moving vehicle. A further goal may be to build a numerical model to describe more precisely the influence of the traffic-induced vibrations on the overall bridge response.

\section{References}

[1] M. Hui, A. Larsen, H. Xiang, Journal of Wind Engineering and Industrial Aerodynamics 97, 48 (2009)

[2] M. Hui, A. Larsen, H. Xiang, Journal of Wind Engineering and Industrial Aerodynamics 97, 22 (2009)

[3] J.H. Macdonald, Tech. rep., Bristol Earthquake and Engineering Laboratory Ltd. (2004)

[4] C. Cai, S. Chen, Journal of Wind Engineering and Industrial Aerodynamics 92, 579 (2004)

[5] IEC, Wind turbine - part 1: Design requirements, iec $61400-1(2005)$

[6] C.Y. Kim, D.S. Jung, N.S. Kim, S.D. Kwon, M.Q. Feng, Earthquake Engineering and Engineering Vibration 2, 109 (2003)

[7] A.G. Davenport, Journal of Structural Division 88, 233 (1962) 